\title{
Experimentos com Programas Lineares por Partes em Redes
}

C. PERIN ${ }^{1}$, M. MELLO ${ }^{2}$, Departamento de Matemática Aplicada, Instituto de Matemática, Estatística e Computação Científica, Universidade Estadual de Campinas, UNICAMP, Cx.P. 6065, 13083-200 Campinas, SP, Brasil

F.A.S. MARINS3, Departamento de Engenharia de Produção, Campus de Guaratinguetá, Universidade Estadual Paulista, UNESP, Cx.P. 205, 12500-000 Guaratinguetá, SP, Brasil.

Resumo. A estratégia usual de resolução de um problema de fluxo em redes lineares por partes é transformá-lo em um problema de fluxo em uma rede equivalente. Neste caso, uma rede linear por partes com $n$ nós e $m$ arcos com $k$ intervalos por arco é convertida em uma rede linear com $n$ nós e $k m$ arcos. Métodos de pontos interiores têm tido um grande sucesso na solução de problemas de fluxo em redes de grande porte devido ao baixo tempo computacional dispendido. Mostramos que é vantajoso especializar o método de pontos interiores para problemas lineares de modo a resolver diretamente problemas de fluxo em redes lineares por partes, ao invés de utilizar sua versão genérica para resolver a forma equivalente de fluxo em redes. Realizamos também testes computacionais para efetuar diversas comparações entre: o uso da estratégia preditor-corretor versus preditor puro, estratégias de inicialização do corretor e dos critérios de parada no preditor e no corretor.

\section{Introdução}

Uma importante área de estudos e aplicações da Programação Matemática é a Programação Linear por Partes que está relacionada com a otimização de uma função objetivo separável, convexa e linear por partes. Neste trabalho, é dada uma atenção especial à sub-área denominada de Programação Linear por Partes em Redes que apresenta relevantes aplicações no mundo real, tais como em: sistemas de potência, redes hidráulicas, transportes, redes de comunicações, administração hídrica, e mesmo problemas estocásticos de fluxo em redes. Já investigou-se [2] a especialização do método simplex para redes para o caso linear por partes com sucesso.

Damos prosseguimento à pesquisa relatada em [6]. Naquele trabalho foi descrita a implementação de uma adaptação do método de pontos interiores para problemas

\footnotetext{
${ }^{1}$ clovis@ime.unicamp.br

2 margarid@ime.unicamp.br

3 fmarins@feg.unesp.br
} 
de fluxo lineares por partes. Esta implementação é agora modificada, incorporando melhorias propostas por [5], especificamente no que se refere à utilização de preditor-corretor. Cabe notar que as implementações usuais do esquema de resolução preditor-corretor de [5] utilizam a decomposição de Cholesky, como, por exemplo, [1, 4]. Na nossa implementação, no entanto, seguimos [7] e resolvemos os sistemas lineares, produzidos pelo esquema preditor-corretor, pelo método de Gradientes Conjugados, que tira proveito da estrutura da matriz (de incidência) de coeficientes do problema, conforme explicitado em [6].

Os algoritmos são descritos na seção 2. Suas implementações são o objeto da seção 3. Os testes computacionais são relatados e comentados na seção 4.

\section{Algoritmos}

O problema de fluxo em redes lineares por partes é definido por

$$
\min \{f(x) \mid A x=b, x \geq 0\}
$$

onde $A$ é a $n \times m$-matriz de incidência de uma rede com $n$ nós e $m$ arcos, $b$ é o $n$ vetor de demandas dos nós, $x$ é um $m$-vetor de fluxos nos arcos, a ser determinado, e $f(x)=\sum_{j=1}^{m} f_{j}\left(x_{j}\right)$ é uma função convexa, separável e linear por partes. As funções convexas e lineares por partes $f_{j}$ podem ser especificadas pelo par de $\ell$-vetores $c, d$, onde $\ell=\sum_{j=1}^{m} \ell_{j}$ é o número total de intervalos, sendo $\ell_{j}$ o número de intervalos de $f_{j}$.

Este problema pode ser transformado, ver [3], em um problema de fluxo em redes lineares definido por

$$
\min \left\{c^{\prime} \bar{x} \mid \bar{A} \bar{x}=b, \bar{x}+\bar{s}=d, \bar{x}, \bar{s} \geq 0\right\} .
$$

Neste caso, a $n \times \ell$-matriz $\bar{A}$ é obtida a partir da matriz $A$ com a replicação de cada uma de suas colunas tantas vezes quantos são os intervalos a ela associados. Estamos supondo que esta replicação também é feita no vetor $x$ transformando-o no vetor $\bar{x}$. O problema dual associado é dado por

$$
\max \left\{b^{\prime} y-d^{\prime} \bar{w} \mid \bar{A}^{\prime} y-\bar{z}+\bar{w}=c, \bar{z}, \bar{w} \geq 0\right\}
$$

e as condições de folgas complementares são expressas por

$$
\bar{X} \bar{Z} 1=0 \quad \text { e } \quad \bar{S} \bar{W} 1=0,
$$

onde $\bar{X}, \bar{S}, \bar{Z}, \bar{W}$ são matrizes diagonais com os elementos de $\bar{x}, \bar{s}, \bar{z}, \bar{w}$, respectivamente, e 1 é um vetor de 1 's.

Foram estudadas duas versões de métodos de pontos interiores: a versão primaldual usual (preditor puro) e a versão primal-dual com preditor-corretor.

Conforme descrito em [6], o método primal-dual usual de pontos interiores é inicializado a partir de um iterando $\bar{x}, \bar{s}, y, \bar{z}, \bar{w}$ satisfazendo $\bar{x}, \bar{s}, \bar{z}, \bar{w}>0$ e a cada 
iteração é obtida uma direção de deslocamento $\Delta \bar{x}, \Delta \bar{s}, \Delta y, \Delta \bar{z}, \Delta \bar{w}$ em conformidade com um parâmetro de centragem $\mu$ de acordo com as equações (2.1) abaixo

$$
\begin{aligned}
\bar{A} \Delta \bar{x} & =b-\bar{A} \bar{x}, \\
\Delta \bar{x}+\Delta \bar{s} & =d-\bar{x}-\bar{s}, \\
\bar{A}^{\prime} \Delta y+\Delta \bar{z}-\Delta \bar{w} & =c-\bar{A}^{\prime} y-\bar{z}+\bar{w}, \\
\bar{X} \Delta \bar{z}+\bar{Z} \Delta \bar{x} & =\mu 1-\bar{X} \bar{z} \\
\bar{S} \Delta \bar{w}+\bar{W} \Delta \bar{s} & =\mu 1-\bar{S} \bar{w},
\end{aligned}
$$

que refletem a factibilidade primal, a factibilidade dual, e a complementaridade das soluções quando $\mu=0$.

O método primal-dual usual de pontos interiores pode ser esquematizado da seguinte forma:

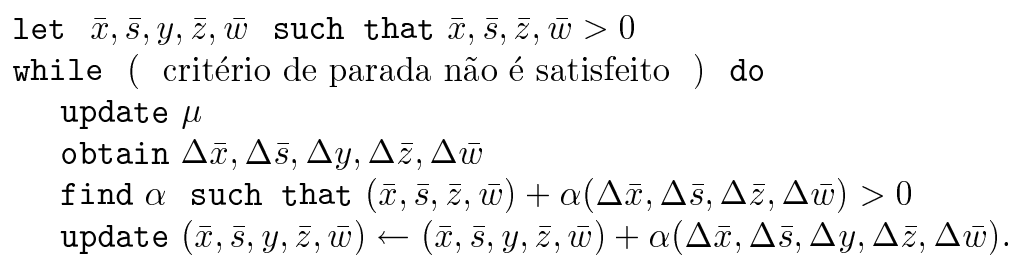

Já no método primal-dual com preditor-corretor são resolvidos dois sistemas: o preditor (2.1) e o corretor (2.2) abaixo, que possui a mesma matriz de coeficientes no lado esquerdo:

$$
\begin{aligned}
\bar{A} \Delta \bar{x} & =b-\bar{A} \bar{x} \\
\Delta \bar{x}+\Delta \bar{s} & =d-\bar{x}-\bar{s}, \\
\bar{A}^{\prime} \Delta y+\Delta \bar{z}-\Delta \bar{w} & =c-\bar{A}^{\prime} y-\bar{z}+\bar{w} \\
\bar{X} \Delta \bar{z}+\bar{Z} \Delta \bar{x} & =\mu 1-\bar{X} \bar{z}-\delta \bar{X} \delta \bar{z} \\
\bar{S} \Delta \bar{w}+\bar{W} \Delta \bar{s} & =\mu 1-\bar{S} \bar{w}-\delta \bar{S} \delta \bar{w}
\end{aligned}
$$

onde $\delta \bar{x}, \delta \bar{z}, \delta \bar{s}$ e $\delta \bar{w}$ correspondem à solução do sistema (2.1).

O vetor $(\Delta \bar{x}, \Delta \bar{s}, \Delta \bar{z}, \Delta \bar{w})$ do algoritmo é obtido pela resolução de (2.1) na versão preditor puro e de (2.2) na versão preditor-corretor. O parâmetro $\alpha$ é obtido por um simples teste da razão na versão preditor puro e de forma especial, como descrito em $[1,5]$.

Utilizamos a estratégia de resolver cada um dos sistemas preditor e corretor por meio da resolução de um $n \times n$-sistema SDP pelo método do gradiente conjugado. A matriz deste sistema é utilizada implicitamente percorrendo os arcos da rede do problema. Estes sistemas SDPs são resolvidos pelo método do gradiente conjugado com precondicionamento diagonal nas primeiras seis iterações do método primaldual e com o precondicionamento da árvore geradora nas iterações subseqüentes. Este procedimento baseia-se em estratégia similar adotada em [7].

Os critérios de parada adotados são: (1) número máximo de iterações é excedido; (2) soluções quase-complementares $\bar{x}^{\prime} \bar{z}+\bar{s}^{\prime} \bar{w} \approx 0$ são encontradas; (3) limite inferior para o valor da função objetivo primal é excedido; (4) limite superior para o valor da função objetivo dual é excedido. 


\section{Implementação}

Foi utilizada a linguagem C na programação dos algoritmos. Foram implementados e testados 4 pares de programas com diferentes versões do método de pontos interiores; 4 para programas em redes lineares e 4 para progamas em redes lineares por partes. Um par (FL9, FP9) é do tipo usual ou preditor puro e os 3 outros (FL0-2, FP0-2) são do tipo preditor-corretor. O sistema corretor é construído de tal forma que a sua solução já componha a direção de deslocamento do iterando. Assim, podemos aplicar o método do gradiente conjugado utilizando a solução do sistema preditor como solução de partida do sistema corretor. A solução de partida do sistema preditor é a solução nula e a solução de partida do sistema corretor é a solução final do primeiro sistema, em $F L 0-1, F P 0-1$, e é a solução nula, no par FL2, FP2. O critério de parada do gradiente conjugado sempre utiliza a tolerância de $10^{-8}$ para o erro relativo da solução corrente no último sistema. Apenas o par FL1, FP1 utiliza a tolerância de $10^{-6}$ para o erro relativo da solução corrente no primeiro sistema. Em resumo, os programas são:

FL9 - preditor puro para redes lineares com tolerância de $10^{-8}$ no erro relativo da solução corrente para terminar o gradiente conjugado.

FLO - preditor-corretor com tolerâncias de $10^{-8}, 10^{-8}$; utiliza a solução final do sistema preditor como solução de partida do sistema corretor.

FL1 - preditor-corretor com tolerâncias de $10^{-6}, 10^{-8}$; utiliza a solução final do sistema preditor como solução de partida do sistema corretor.

FL2 - preditor-corretor com tolerâncias de $10^{-8}, 10^{-8}$; utiliza a solução nula na partida do sistema corretor.

FP9 - preditor puro para redes lineares por partes com tolerância de $10^{-8}$ no erro relativo da solução corrente para terminar o gradiente conjugado.

FP0 - preditor-corretor com tolerâncias de $10^{-8}, 10^{-8}$.

FP1 - preditor-corretor com tolerâncias de $10^{-6}, 10^{-8}$.

FP2 - preditor-corretor com tolerâncias de $10^{-8}, 10^{-8}$; utiliza a solução nula na partida do sistema corretor.

O espaço de memória requerido por estes programas para armazenar os dados da rede, das soluções correntes, da aplicação do gradiente conjugado e de outras variáveis auxiliares pode ser resumido em: (FL9) $13 n$-vetores e $16 \ell$-vetores; (FL0-2) $15 n$-vetores e $19 \ell$-vetores; (FP9) $13 n$-vetores, $4 m$-vetores e $13 \ell$-vetores; (FP0-2) $15 n$-vetores, $4 \mathrm{~m}$-vetores e $16 \ell$-vetores.

As tolerâncias utilizadas nos critérios de parada são: $10^{-8}$ (para tolerância relativa no teste de complementaridade), $-10^{8}$ (para limite inferior da função objetivo primal) e $10^{8}$ (para limite superior da função objetivo dual). 


\section{Testes Computacionais}

Os programas foram testados em um micro computador Pentium III, $500 \mathrm{MHz}$ de clock e $328 \mathrm{MB}$ de memória RAM utilizando problemas em redes conectadas geradas aleatoriamente com até 100 mil nós, 1 milhão de arcos e 1 milhão de intervalos. As redes correspondem a problemas de transporte modificados. À estrutura de digrafo bipartido com igual número de nós de oferta e de demanda acrescentou-se um ciclo orientado contendo os nós de oferta e outro contendo os nós de demanda, com o objetivo de tornar os problemas gerados factíveis. Todos os nós das redes geradas apresentam o mesmo grau. As ofertas/demandas, os coeficientes de custos e os coeficientes de capacidades foram geradas com distribuição uniforme em intervalos fornecidos como parâmetros do programa gerador. As tabelas a seguir contêm os tempos de CPU (em segundos), o número de iterações do método primal-dual e o número total de iterações do método do gradiente conjugado executados em cada programa. Estes dados foram colhidos em 5 testes para cada configuração de rede (mesmo conjunto de parâmetros e sementes diferentes) e as médias dos 5 valores obtidos nestes testes arredondadas para o inteiro mais próximo.

Exemplares iniciais. Primeiramente foram testados exemplares de programas relativamente pequenos, variando os parâmetros fornecidos para o gerador procurando definir direções para este estudo. Os parâmetros envolveram os intervalos de geração das ofertas/demandas nos nós, os intervalos de geração dos ceoficientes de custo, os intervalos de geração das capacidades, o número de nós, o número de arcos e o número de intervalos. Os dados considerados mais promissores correspondem aos exemplares de problemas da Tabela 1 com ofertas/demandas, custos e capacidades gerados aleatoriamente com distribuição uniforme no intervalo $(0,100)$.

A parte superior da Tabela 1 contém as dimensões das redes e sua parte inferior apresenta os tempos de CPU obtidos com os 9 grupos de exemplares que foram resolvidos com as oito versões. Estes dados são também apresentados sob forma gráfica na Figura 1 (FL0, FL1, FL2, FP0, FP1 e FP2) e na Figura 2 (FL9, FL0, FP9 e FP0). Pode-se constatar o desempenho superior das versões especializadas para redes lineares por partes. Em cada uma das classes 0-2 de preditor-corretor as versões do tipo 1 (FP1, FL1) são ligeiramente superiores às demais (vide Figura1). Na Figura 2 pode-se verificar que apenas no grupo G9 a versão preditor-corretor (aqui representada pela implementação $F L 0, F P 0$ ) deixa de ser superior em relação à versão preditor puro FP9.

Variando o número de intervalos. A parte superior da Tabela 2 apresenta os tempos de CPU obtidos com os oito programas em exemplares gerados com 10000 nós, 35000 arcos e número intervalos variando entre 70000 e 1050000 . Estes dados constatam um desempenho superior das versões especializadas para redes lineares por partes. Além disto, o programa FP9 apresenta-se melhor do que os demais para quase todos os exemplares (vide Figura 3).

Variando o número de arcos. A parte central da Tabela 2 apresenta os tempos de CPU obtidos com os oito programas em exemplares gerados com 10000 


\begin{tabular}{c|rrr|rrr|rrr}
\hline Grupos & G1 & G2 & G3 & G4 & G5 & G6 & G7 & G8 & G9 \\
\hline \# nós $\left(10^{3}\right)$ & 10 & 10 & 10 & 10 & 10 & 10 & 10 & 10 & 10 \\
\# arcos $\left(10^{3}\right)$ & 20 & 20 & 20 & 35 & 35 & 35 & 50 & 50 & 50 \\
\# inter. $\left(10^{3}\right)$ & 40 & 100 & 160 & 70 & 105 & 280 & 100 & 250 & 400 \\
\hline FL9 & 988 & 859 & 772 & 913 & 674 & 636 & 857 & 649 & 775 \\
FL0 & 175 & 295 & 468 & 301 & 655 & 1014 & 447 & 1002 & 1576 \\
FL1 & 169 & 280 & 447 & 291 & 640 & 980 & 432 & 980 & 1613 \\
FL2 & 186 & 313 & 502 & 322 & 721 & 1085 & 479 & 1097 & 1740 \\
FP9 & 846 & 637 & 513 & 744 & 456 & 369 & 670 & 409 & $\mathbf{3 9 8}$ \\
FP0 & 125 & 168 & 205 & 200 & 281 & 358 & 284 & 425 & 533 \\
FP1 & $\mathbf{1 2 0}$ & $\mathbf{1 5 4}$ & $\mathbf{1 8 6}$ & $\mathbf{1 9 2}$ & $\mathbf{2 5 8}$ & $\mathbf{3 1 8}$ & $\mathbf{2 5 6}$ & $\mathbf{3 6 7}$ & 478 \\
FP2 & 130 & 165 & 197 & 202 & 274 & 338 & 281 & 398 & 498 \\
\hline
\end{tabular}

Tabela 1: Dimensões das redes e segundos de CPU dos exemplares iniciais, com tempos mínimos em negrito

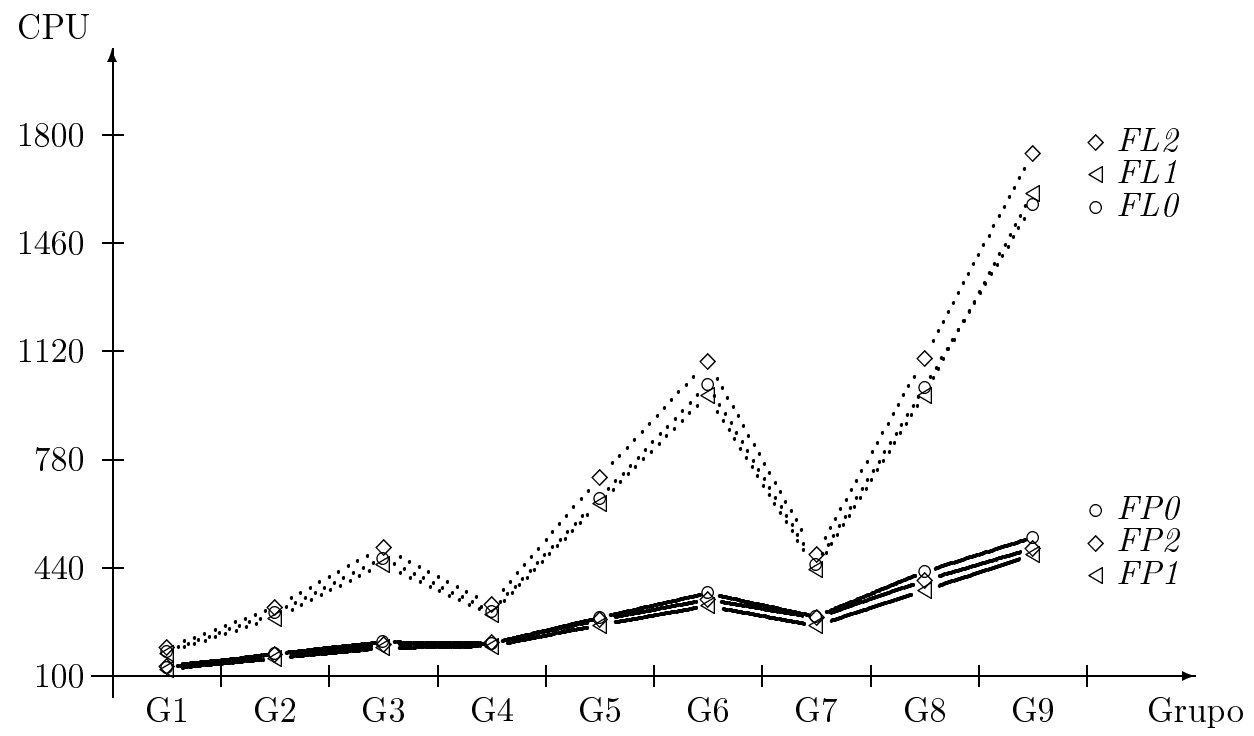

Figura 1: Segundos de CPU para as versões do tipo preditor-corretor 


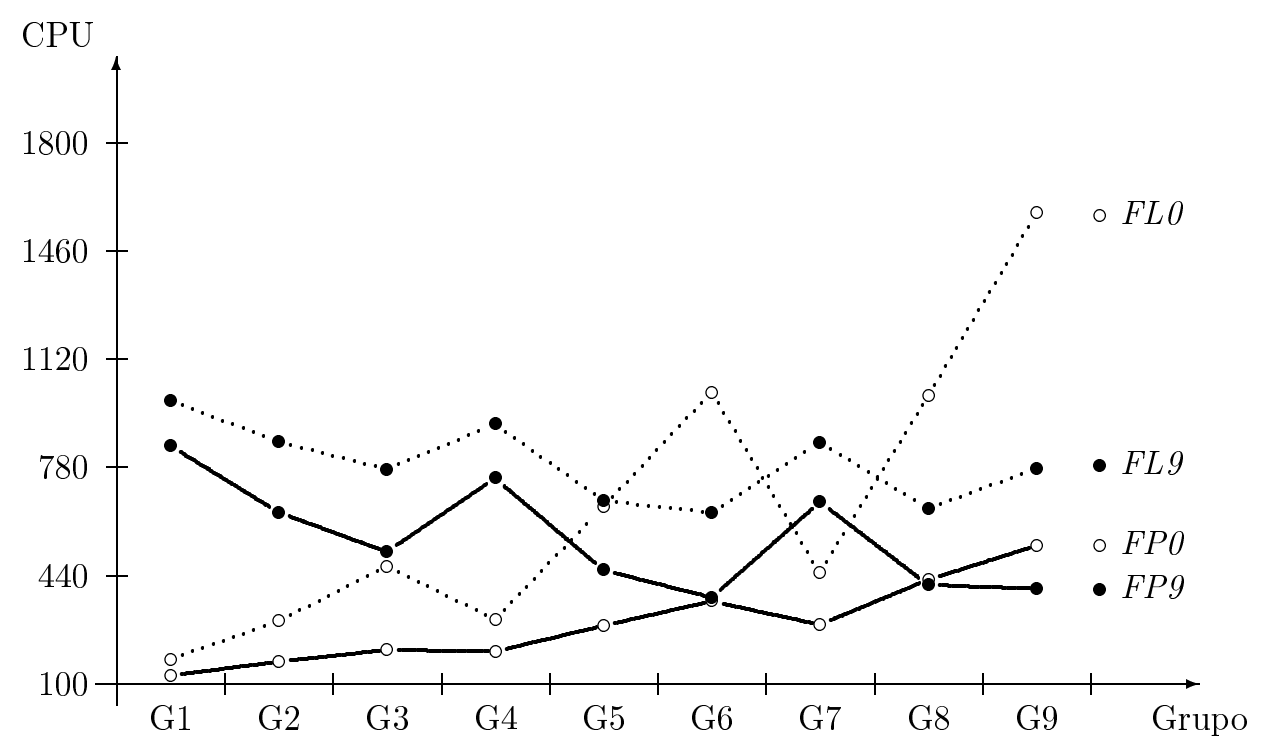

Figura 2: Segundos de CPU para FL9, FLO, FP9 e FPO

\begin{tabular}{|c|c|c|c|c|c|c|c|c|}
\hline Prog & FL9 & FLO & $F L 1$ & FL2 & FP9 & FPO & FP1 & $F P^{2}$ \\
\hline intervalos & \multicolumn{8}{|c|}{10000 nós, 35000 arcos } \\
\hline $70 \mathrm{mil}$ & 913 & 301 & 291 & 322 & 744 & 200 & 192 & 202 \\
\hline 175 mil & 674 & 655 & 640 & 721 & 456 & 281 & 258 & 274 \\
\hline $280 \mathrm{mil}$ & 636 & 1014 & 980 & 1085 & 369 & 358 & 318 & 338 \\
\hline $525 \mathrm{mil}$ & 877 & 1784 & 1918 & 1995 & 382 & 461 & 457 & 446 \\
\hline $1050 \mathrm{mil}$ & 1806 & 4125 & 4390 & 4640 & 529 & 702 & 687 & 705 \\
\hline $\operatorname{arcos}$ & \multicolumn{8}{|c|}{10000 nós, 5 intervalos/arco } \\
\hline $20 \mathrm{mil}$ & 859 & 305 & 280 & 313 & 637 & 161 & 154 & 165 \\
\hline $35 \mathrm{mil}$ & 674 & 697 & 640 & 721 & 456 & 271 & 258 & 274 \\
\hline $50 \mathrm{mil}$ & 649 & 1077 & 980 & 1097 & 409 & 398 & 367 & 398 \\
\hline 85 mil & 913 & 2179 & 1997 & 2259 & 485 & 736 & 675 & 746 \\
\hline $160 \mathrm{mil}$ & 1970 & 5389 & 4978 & 5746 & 878 & 1587 & 1471 & 1647 \\
\hline nós & \multicolumn{8}{|c|}{50000 arcos, 250000 intervalos } \\
\hline 5 mil & 334 & 798 & 737 & 832 & 165 & 248 & 236 & 249 \\
\hline 10 mil & 649 & 1077 & 980 & 1097 & 409 & 398 & 367 & 398 \\
\hline $12.5 \mathrm{mil}$ & 970 & 1171 & 1090 & 1200 & 650 & 472 & 442 & 480 \\
\hline 20 mil & 3120 & 1450 & 1339 & 1496 & 2371 & 735 & 695 & 739 \\
\hline
\end{tabular}

Tabela 2: Segundos de CPU com variação de parâmetros, com mínimos em negrito 


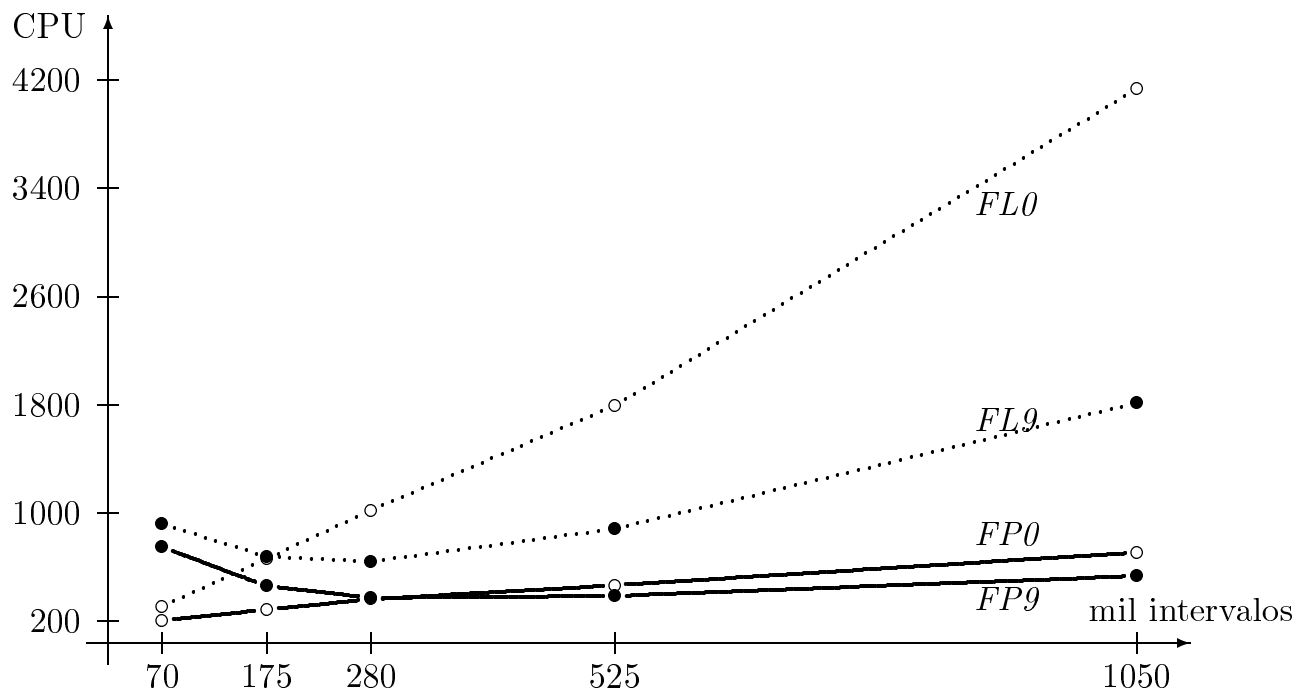

Figura 3: Segundos de CPU variando o número de intervalos

nós e número de arcos variando entre 20000 e 160000. Estes dados constatam um desempenho superior das versões especializadas para redes lineares por partes. Além disto, o programa FP9 apresenta-se melhor do que os demais para quase todos os exemplares (vide Figura 4).

Variando o número de nós. A parte inferior da Tabela 2 apresenta os tempos de CPU obtidos com os oito programas em exemplares gerados com 50000 arcos, 250000 intervalos e número de nós variando de 5000 a 20000. Estes dados constatam um desempenho superior das versões especializadas para redes lineares por partes. Nestes testes, as versões do tipo preditor-corretor, em especial FP1, apresentam-se com um comportamento superior aos das demais versões (vide Figura 5).

\section{Comentários Finais}

Nos experimentos foram também contabilizados o número de iterações do método primal-dual e o número total de iterações do método do gradiente conjugado com precondicionamento diagonal e com precondicionamento da árvore geradora máxima. De um modo geral, o número de iterações do método primal-dual na versão usual ou preditor puro foi superior a 2 vezes o número de iterações do método primal-dual nas versões preditor-corretor. Verificou-se que seria melhor utilizar um critério para número de iterações com precondicionador diagonal que dependesse do tamanho da rede (nas redes maiores o precondicionador diagonal seria utilizado em mais do que 6 iterações iniciais). Entre as versões preditor-corretor não houve diferença significativa no número de iterações do método primal-dual. Outros tipos de variações na estrutura e nos coeficientes das redes foram testados; selecionamos para apresentação neste artigo os resultados considerados mais interessantes.

Em resumo, a versão preditor-puro parece ter um comportamento melhor apenas 


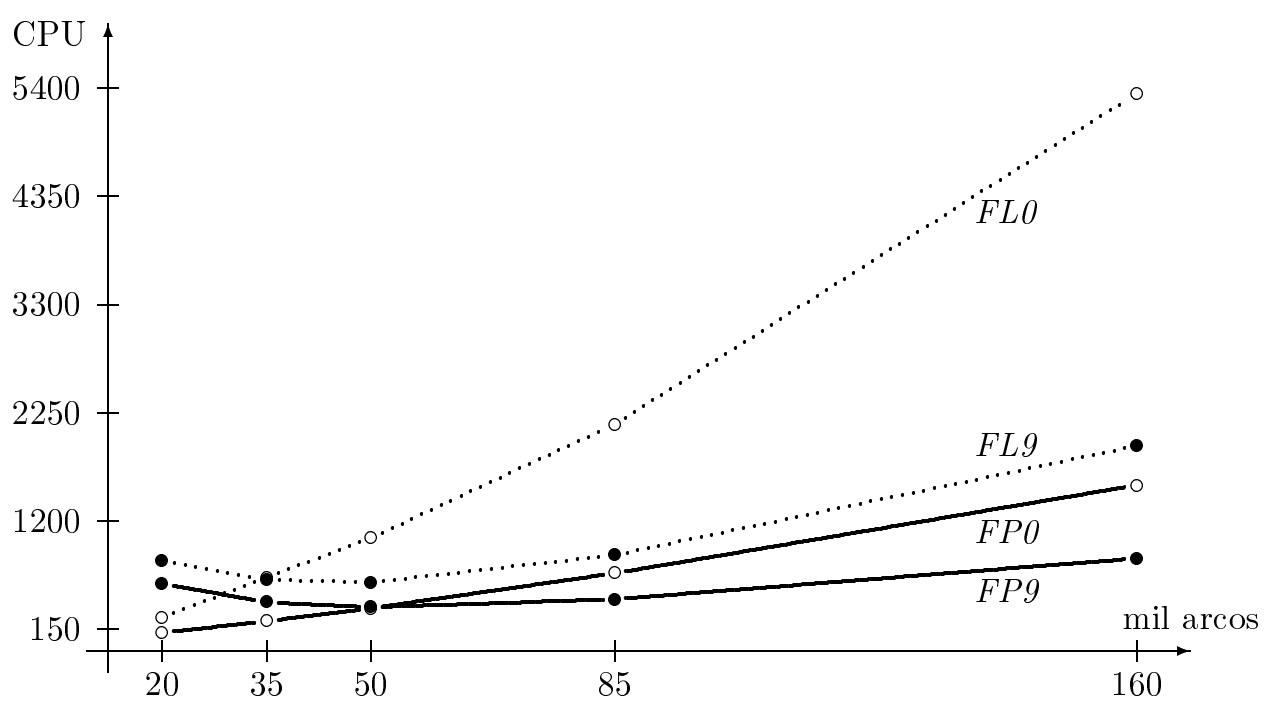

Figura 4: Segundos de CPU variando o número de arcos

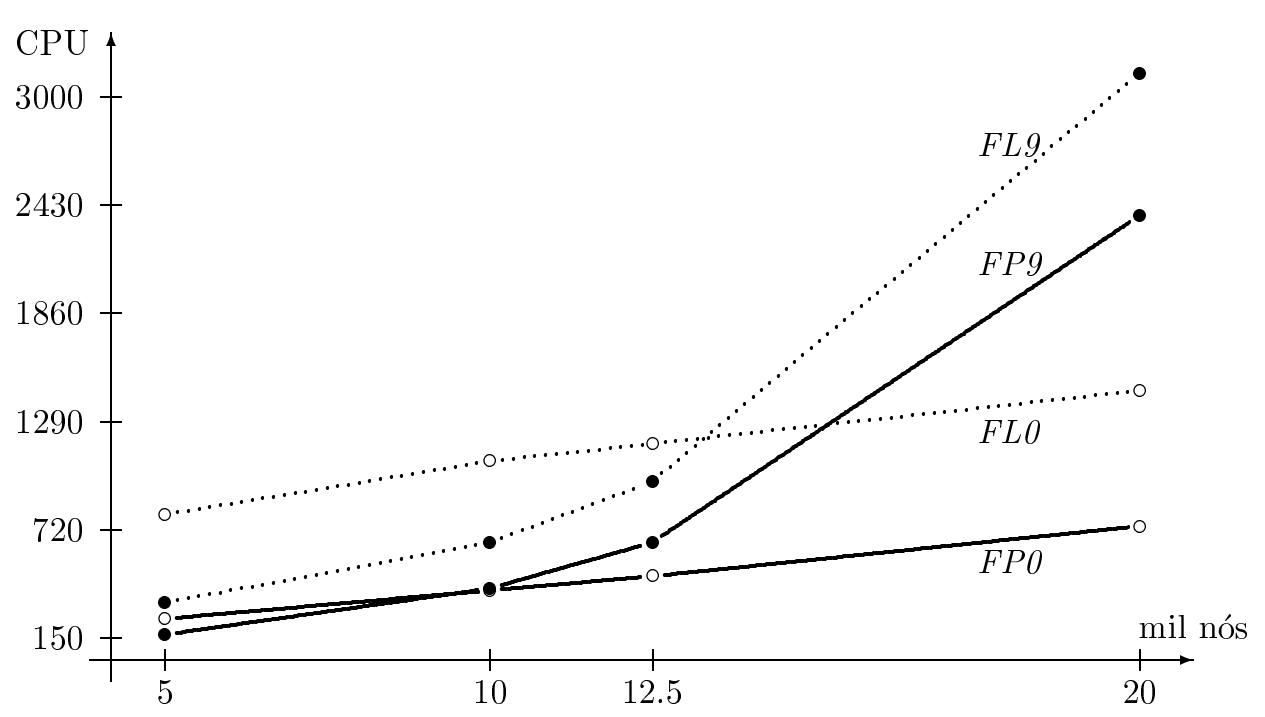

Figura 5: Segundos de CPU variando o número de nós 
quando a rede tem um grande número de arcos ou intervalos em comparação com o número de nós.

\begin{abstract}
The usual approach to solving a piecewise linear network flow problem is to transform it into an equivalent linear one. In this transformation, a piecewise network with $n$ nodes and $m$ arcs, each with $k$ intervals (corresponding to the linear pieces of the arc cost function), has an equivalent linear one with $n$ nodes and $m k$ arcs. Interior point methods have been proved successful in the solution of linear network flow problems. We show that it is advantadgeous to construct a customized interior point method to solve piecewise network problems directly, instead of applying its generic version to the equivalent linear problem. Two algorithms were implemented and tested: one using predictor-corrector and the other without the corrector step. Comparison between alternative strategies (initialization, stopping criteria) are done by means of several computacional tests.
\end{abstract}

\title{
Referências
}

[1] J. Czyzyk, S. Mehrotra, M. Wagner e S.J. Wright, PCx user guide, Technical Report OTC 96/01, Optimization Technology Center, 1997.

[2] K. Darby-Dowman, F.A.S. Marins, E. Senne, C. Perin e A. Machado, Algorithms for network piecewise-linear programs: a comparative study, European Journal of Operational Research 97 (1997), 183-199.

[3] J.K. Ho, Relationships among linear formulations of separable convex piecewise linear programs, Mathematical Programming Study 24 (1985), 126-140.

[4] M. Kojima, N. Megiddo e S. Mizuno, A primal-dual infeasible-interior-point algorithm for linear programming, Mathematical Programming 61 (1993), 263280 .

[5] S. Mehotra, On the implementation of a primal-dual interior point method, SIAM Journal on Optimization 2 (1989), 575-601.

[6] C. Perin, M.P. Mello e F.A.S. Marins, Uma implementação de pontos interiores para fluxo em redes lineares por partes, in "Seleta do XXII CNMAC", Tendências em Matemática Aplicada e Computacional, Vol. 1, Parte 2, pp. 431-442, SBMAC, 2000.

[7] M. Resende e G. Veiga, An efficient implementation of a network interior point method, Technical Report, AT\&T Bell Laboratories, Murray Hill, NJ, USA, 1992. 